\title{
An Aerial Weed Detection System for Green Onion Crops Using the You Only Look Once (YOLOv3) Deep Learning Algorithm
}

\author{
Addie Ira Borja PARICO ${ }^{1}$, Tofael AHAMED ${ }^{2 *}$
}

\begin{abstract}
The real-time object detection system You Only Look Once (specifically YOLOv3) has recently shown remarkable speed, making it potentially suitable for Unmanned Aerial Vehicle (UAV) precision spraying. In this study, YOLO-WEED, a weed detection system based on YOLOv3, was developed. The dataset, derived from a five-minute UAV video, was split into a $69: 17: 13$ ratio for training, validation, and testing, respectively. YOLO-WEED demonstrated a real-time detection speed (up to 24.4 FPS) and high performance using NVIDIA GeForce GTX 1060, with a mean average precision of $93.81 \%$ and an F1 score of 0.94 . These results successfully show the effectiveness of the YOLO-WEED system for real-time UAV weed detection, given its high speed and high accuracy in detection.
\end{abstract}

[Keywords] you only look once (YOLO), deep learning, real time weed detection, convolutional neural network (CNN), unmanned aerial vehicle

\section{Introduction}

Weed control is very important for green onion crops as weeds can easily outcompete and grow more rapidly, thus, shading the crop and competing for nutrients and water (FDA, 2016; Gilreath et al., 2008; Hewson and Roberts, 1973). The largest expenditures in green onion production in terms of pest management, after all, come from weed control (Norsworthy et al., 2007). In research progress of precision application with site-specific weed control, herbicide use can be reduced by an average value of $54 \%$ (Timmermann et al., 2003).

Still, farmers may have hesitations in adopting site-specific weed control measures if they need to spend more for equipment and data processing. To close this cost gap, unmanned aerial vehicles (UAVs), a more cost-effective remote sensing platform, is gaining momentum in precision spraying in the past decade. Recently, UAVs are becoming more technologically advanced and affordable. In addition, compared to other remote sensing platforms, UAVs are most advantageous in terms of maneuverability and resolution (for temporal and spatial) (Tsouros et al., 2019). Moreover, UAV sprayers can be more suitable to the small scale farmers in Japan compared to the conventional large-scale boom sprayers (He et al., 2017).

Real time detection of weeds is necessary in utilizing UAV for precision spraying (Lan et al., 2010). You Only Look Once (YOLO), a neural network-based object detection algorithm, has attracted attention from the deep learning field due to its remarkable speed, boasting an inference time range of 7 to
$46 \mathrm{~ms}$ for its YOLOv3 version (Koirala et al., 2019; Kuznetsova et al., 2020; Lawal, 2021). Czymmek et al. (2019) has utilized a modified YOLO approach in real-time detection of weeds using a multiplatform robot. Only 50 images are used for training dataset and achieved a detection speed of 56 FPS, however, had a lower accuracy as the trade-off with speed. Other studies have examined the detection performance of tiny YOLO, a lighter version, on weed detection too but had the same results: faster speed entails lower accuracy (Gao et al., 2020; Partel et al., 2019; Zhang et al., 2018). There are no studies done yet regarding the performance of YOLOv3 using aerial videos for weeds detection and recognition accuracy. Thus, in this study, a YOLOv3-based weed detection system, which is called YOLO-WEED from hereon, was developed and evaluated using a UAV video as the source of dataset.

\section{Materials and Methods}

\section{Data acquisition}

Video acquisition was done in a green onion field with broad-leaf weed incidences in Yatabe, Tsukuba-shi, Ibaraki-ken, Japan $\left(36^{\circ} 00.414^{\prime} \mathrm{N}, 140^{\circ} 05.349^{\prime} \mathrm{E}\right)$ on a clear day on May 2019 using DJI Phantom 3 Pro (DJI Co. Ltd., China). The flight plan is illustrated in Fig. 1. The video, having the resolution of $4096 \times 2160$, was approximately $5 \mathrm{~min}$ long, recorded at $4-5 \mathrm{~m}$ altitude at a very slow speed $(0-1.3 \mathrm{~m} / \mathrm{s})$.

\section{Dataset preparation and augmentation}

The video was divided into two parts: first $4 \mathrm{~min} 30 \mathrm{~s}$ and

${ }^{1}$ Graduate School of Life and Environmental Sciences, University of Tsukuba, Japan

${ }^{2}$ Faculty of Life and Environmental Sciences, University of Tsukuba, Japan

*Corresponding author: tofael.ahamed.gp@u.tsukuba.ac.jp 


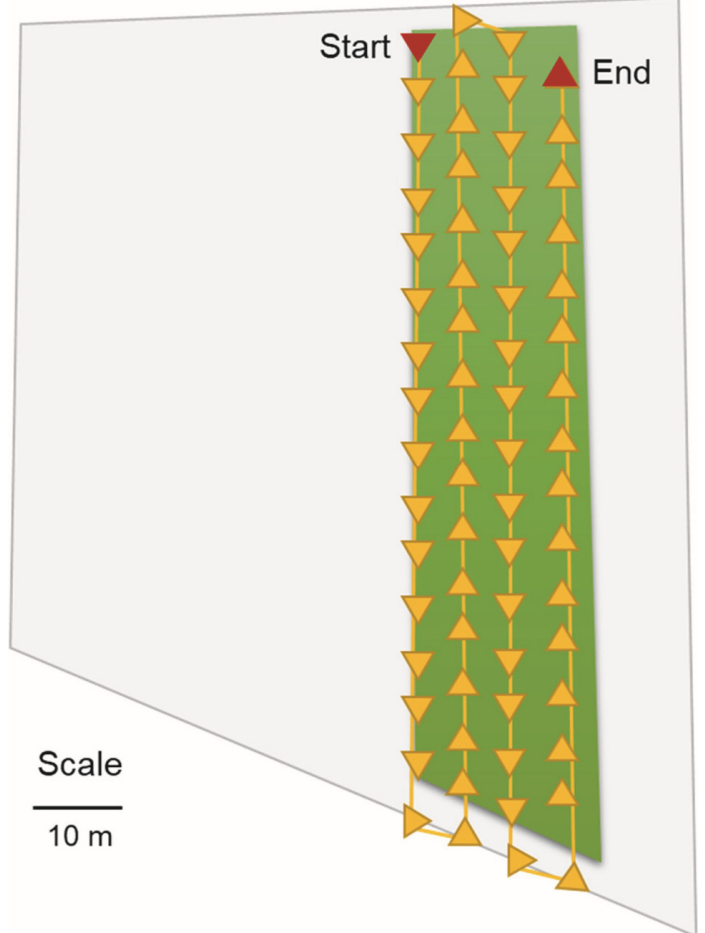

Fig. 1 Flight path for video acquisition of green onion with weeds using an unmanned aerial vehicle at $4-5 \mathrm{~m}$ altitude at $0-1.3 \mathrm{~m} / \mathrm{s}$ speed

the last $30 \mathrm{~s}$. The second part was set aside for testing the real-time performance of YOLO-WEED. The first part was divided into image frames by using VLC to capture the video frames per second ( 1 capture for every 24 frames). Each screen captures were manually inspected to make sure the screen captures are unique from each other. Then, all unique screen captures were divided into 96 tiles ( 8 rows, 12 columns) to reduce human errors in identifying the weeds. Total of 520 images (size $341 \times 270$ pixels) with both green onion and weeds of differing exposure, clarity, incidence and object sizes were generated.

To reduce overfitting, the dataset was artificially enlarged through data augmentation. The intensities of the RGB channels were altered through Principal Component Analysis (PCA) color augmentation (Krizhevsky et al., 2017). This data augmentation was chosen because the color offset preserves the identity of the object of interest despite modification in the intensity and color of the illumination (Bargoti and Underwood, 2016; Krizhevsky et al., 2017). PCA color augmentation was implemented based on the method by Krizhevsky et al., (2012). PCA-based color augmentation was used to simulate different color balances resulting from different conditions of illumination.

After PCA color augmentation, 520 perturbed images were generated, resulting total number of images was 1040. The image dataset was split into three sets: training (720 images),
Table 1 The performance metrics that were used to evaluate YOLO-WEED, a YOLOv3-based weed detection system using bounding boxes, are Intersection over Union (IoU), Recall, Precision and F1 score

$\begin{aligned} \text { Performance Metrics } & \text { Equation } \\$\[ o U=\frac{A_{\mathrm{o}}}{A_{\mathrm{u}}} \]$ & =\frac{T P}{T P+F N} \\ P & =\frac{T P}{T P+F P} \\ F 1 & =\frac{2 \times P \times R}{P+R} \\ m A P & =\int_{0}^{1} P(R) d R\end{aligned}$

This evaluation is based on the Pascal VOC Challenge Everingham (Everingham et al., 2010).

$I o U$ : Intersection over Union, $A_{\mathrm{o}}$ : area of overlap, $A_{\mathrm{u}}$ : area of union, $R$ : Recall, TP: True Positive, FP: False Positive, $F N$ : False Negative, $P$ : Precision, $F 1$ : F1 score, $m A P$ : mean Average Precision.

validation (180 images) and testing (140 images). Weeds in these images were labeled with an open-source software BBox-Labeling-Tool (Qiu and Shrivastava, 2017). Then, the generated labels were converted into YOLO format.

\section{Weed detection with YOLOv3 algorithm}

To set up the weed detection system, Darknet, the neural network framework of YOLOv3, originally authored by Redmon and Farhadi (2018), was used from a stable and improved Github repository of Darknet (https://github.com/AlexeyAB /darknet). GPU-enabled YOLOv3 (Redmon and Farhadi, 2018) and compiled in Windows 10 operating system using Visual Studio with the dependencies installed (CUDA version 10.1.168, OpenCV 3.4.0, cuDNN 10.1). The hardware used had the following specifications: Quad-core Intel Core i77700HW@2.80 GHz (Intel Corp., USA), NVIDIA GeForce GTX 1060 and 16 GB RAM (NVIDIA Corp., USA).

(1) Metrics for evaluating the performance of YOLOv3

The performance of YOLOv3 was evaluated based on the metrics used in the Pascal VOC Challenge (Everingham et al., 2010), which are listed in Table 1 . The first metric is Intersection over Union $(I o U)$, which is the ratio between the area of overlap and the area of union of the bounding boxes of the prediction and the ground truth object.

To calculate the other performance metrics, true positive, false positive and false negative detections should be deter- 
mined first. For a detection to be considered as True Positive $(T P)$, or ground truth objects that were correctly identified, $I o U$ should be equal to or greater than 0.5 . It was deliberately set this low to account for human errors in creating the bounding boxes for the ground truth. For example, if a plant has radial stems but most of its vegetative parts are at the center, is somewhat subjective. False positive $(F P)$ detections, on the other hand, are those having $I o U$ values under 0.5 . Finally, false negative $(F N)$ detections were the ground truth objects that were completely missed by the predictions or those assigned with low confidences in predictions (eliminated by a certain threshold, which was considered 0.25 in this research).

After calculating $T P, F P$ and $F N$, the following performance metrics can be calculated to determine recall, precision, F1 score and mean average precision. Recall is the sensitivity of the weed detection system. This metric defines the proportion of true positive detections to total ground truth objects. Precision is the proportion of the true positive detections to all positive detections. Next is F1 score, which quantifies the overall performance of detection by incorporating both precision and recall. Finally, mean average precision $(m A P)$ is the area under the precision-recall curve. It is an alternate metric to F1 score in terms of summarizing precision and recall. This metric is often used during the training to select which weights fit the model.

(2) Training YOLOv3

Training is the process where YOLOv3 algorithm fits the training dataset to a predictive model in identifying weeds from images. Default configurations intended specifically for custom object detection for initial training of YOLOv3 (Redmon et al., 2019) were set initially with the pre-trained weights for darknet53 (Redmon, 2016). For each 100 iterations, weights were generated during the training process. Early stopping method was done to reduce the chance of overfitting the model.

YOLOv3 was trained using the loss function below to simultaneously predict whether the weed objects were detected together with the ground truth bounding boxes in the images. The first and second terms of the loss function was calculated for the localization loss of detected objects, which was the error in the predicted bounding box locations and sizes. The third term of the loss function was the confidence loss of the detected object, which measured how likely it was for the bounding box to contain an object of a specific class. The fourth term of the loss function was the confidence loss if no object was detected, where the threshold $\left(\lambda_{\text {noobj }}\right)$ reduced unnecessary detection of objects in the background. Finally, the last term was the classification loss, which, in this study, is not applicable because there were only one object class (weed).

During the training, the mAPs and loss function chart were enabled and monitored. The early stopping point for the training was conducted when the average loss has no longer decreased as much after many iterations and when the highest mAP was achieved. For this case, maximum iteration number of 2000 was implemented.

$$
\begin{aligned}
\text { loss }= & \lambda_{\text {coord }} \sum_{i=0}^{S^{2}} \sum_{j=0}^{B} \mathbb{1}_{i j}^{o b j}\left[\left(x_{i}-\hat{x}_{i}\right)^{2}+\left(y_{i}-\hat{y}_{i}\right)^{2}\right] \\
& +\lambda_{\text {coord }} \sum_{i=0}^{S^{2}} \sum_{j=0}^{B} \mathbb{1}_{i j}^{o b j}\left[\left(\sqrt{w_{i}}-\sqrt{\widehat{w}_{i}}\right)^{2}+\left(\sqrt{h_{i}}-\sqrt{\hat{h}_{i}}\right)^{2}\right] \\
& +\sum_{i=0}^{S^{2}} \sum_{j=0}^{B} \mathbb{1}_{i j}^{o b j}\left(C-\hat{C}_{i}\right)^{2} \\
& +\lambda_{\text {noobj }} \sum_{i=0}^{S^{2}} \sum_{j=0}^{B} \mathbb{1}_{i j}^{n o o b j}\left(C-\hat{C}_{i}\right)^{2} \\
& +\sum_{i=0}^{S^{2}} \mathbb{1}_{i}^{o b j} \sum_{c \in \text { classes }}\left(p_{i}(c)-\hat{p}_{l}(c)\right)^{2}
\end{aligned}
$$

where $\mathbb{1}_{i}^{o b j}$ is when an object appears in cell $i, \mathbb{1}_{i j}^{o b j}$ is when the $j$ th bounding box predictor in cell $i$ is "responsible" for that prediction.

(3) Validation and testing YOLOv3

The purpose of validation is to evaluate the performance of the weights generated from the training. Testing is to make sure overfitting is minimized, which means the YOLO-WEED using the generated weights can also detect weeds from other datasets. Validation and testing in this research were based on the bounding box evaluation of Pascal VOC Challenge (Everingham et al., 2010). Testing was divided into two parts: testing with test images and with the $864 \times 688$ resolution UAV video.

\section{Results and Discussion}

\section{Training YOLOv3 for weed detection}

YOLOv3 was trained with 720 annotated images to detect instances of weeds. The training time was $5 \mathrm{~h}$ and $3 \mathrm{~min}$ using GeForce GTX 1060. Weights were generated every 100 iterations during training. Training was stopped at iteration of 2000 to avoid overfitting. Weights with the lowest loss and highest mAP after the early stopping point (iteration $=1000$ ) were selected for further validation. As seen in Fig. 2, highest mAPs and lowest losses were achieved at iterations 1600 , 1900 and 2000. 
PARICO, AHAMED : An Aerial Weed Detection System for Green Onion Crops

Using the You Only Look Once (YOLOv3) Deep Learning Algorithm

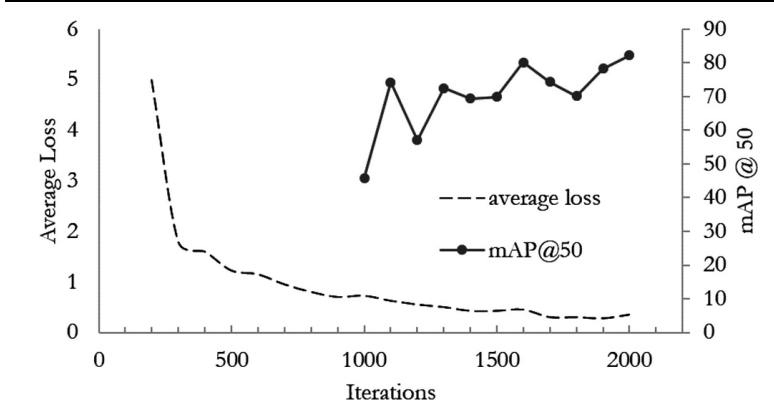

Fig. 2 Loss function and mean average precision values (at $50 \%$ threshold for IoU) during the training at network resolution of $416 \times 416$, learning rate $=0.001$, decay $=0.0005$, momentum $=0.0005$ and batch size $=64$

\section{Validating YOLO-WEED}

The weights were validated using the performance metrics mentioned in Table 1. From the training, weights from iterations 1600, 1900 and 2000 had the highest performance. Based on Fig. 3, scores in iterations 1600 and 2000 are comparable. For iteration 1600, the Average IoU, mAP, Precision, Recall and F1 are 69.8 and $80.2 \%, 0.95,0.73$ and 0.82 , respectively. For iteration 2000, the said scores are just slightly higher, which are 70.4 and $82.2 \%, 0.96$ and 0.84 , respectively. Thus, weights from iteration 2000 were deemed to be most suitable for use. In addition to this, detection speed at the iteration 2000 was $23.7 \mathrm{FPS}$, which is suitable for standard frame rates of videos (24 FPS).

\section{Testing YOLO-WEED}

Well-performing detection in validation may reflect overfitting, therefore, final testing was done with a separate set of 140 annotated images. It is confirmed with high scores in performance metrics that overfitting was minimized at iteration of 2000 (Table 2).

In terms of F1-score and mAP@50, it can be construed that the weed detection system is well-performing in comparison with YOLOv3 and YOLOv2, respectively, on official datasets at $416 \times 416$ network resolution: mAP of 53.2 percent using COCO Dataset (Zhao and Li, 2020) and mAP@50 of 78.6\% using PASCAL VOC 2007 dataset (Redmon and Farhadi, 2017). However, the YOLO-WEED was only trained with one class, which explains its better metrics compared to YOLOv3 being tested with COCO and PASCAL VOC 2007 datasets. Moreover, comparing the mAP@50 with the study by Czymmek et al., (2019), which only used 50 images as dataset, only had an mAP@50 is $53.61 \%$ at an image input size of $416 \times 416$. Comparing it with this, YOLO-WEED's mAP@50 is 1.7 times higher, which may be mainly due to a higher number of training images.

Precision had a very high value of 0.96 , which signifies that

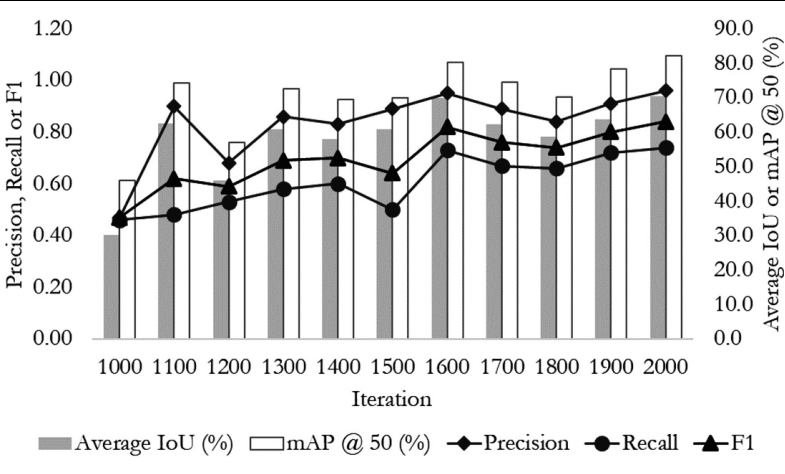

A threshold of $50 \%$ for the IoU (Intersection over Union) is applied for the mAP (mean average precision).

Fig. 3 Validation results among weights from iterations on and after the early stopping point (iteration $=1000$ )

Table 2 The performance of YOLO-WEED, a YOLOv3-based weed detection system, in terms of Average Intersection over Union (IoU), Precision, Recall, Mean Average Precision (at $50 \%$ confidence threshold), and F1 score, with 140 test annotated images using weights from iteration $=2000$ at network resolution of $416 \times 416$

\begin{tabular}{lc}
\hline & Value \\
\hline Average IoU & $72.52 \%$ \\
Precision & 0.96 \\
Recall & 0.92 \\
F1-score & 0.94 \\
mAP@50* & $93.81 \%$ \\
\hline
\end{tabular}

*at $50 \%$ threshold for IoU.

a remarkable amount of the total positive detections were true positive detections. This means that YOLOv3 was able to distinguish green onion from weeds, thus, very little false positive predictions were generated. Recall was relatively lower than precision but still considerably high, which implies that $8 \%$ of the ground truth objects were not detected at all. This is possibly due to the fact that YOLO is disadvantageous in detecting small objects ( $\mathrm{Du}, 2018$ ). It is also possible that there were not enough weed objects during the training that were occluded, thus, detection of occluded weeds may have lower confidence and were eliminated by the confidence threshold.

\section{Testing YOLO-WEED with a UAV video}

As mentioned in Section II, a 5 min aerial video at altitude of $4-5 \mathrm{~m}$ was recorded over the green onion field infested with broad-leaf weeds. The video was split into two parts: the preceding $4 \mathrm{~min}$ and $30 \mathrm{~s}$ and the last $30 \mathrm{~s}$. In this section, the latter part was used to test the real-time performance of 


\section{YOLO-WEED}

First, YOLO-WEED was tested with the $30 \mathrm{~s}$ video at the original resolution of $4096 \times 2160$. However, small weeds were not successfully detected. One reason may be due to the spatial constraint of YOLO in detecting extremely small objects ( $\mathrm{Du}, 2018)$. Second is the implementation of the same value for threshold for all object sizes. In this paper, a threshold of 0.5 was implemented for IoU. The problem is that small objects' IoU can deviate largely with just a slight difference in pixels between the ground truth and prediction (Russakovsky et al., 2015). For example, consider an object of size $10 \times 10$ pixels with a corresponding detection bounding box of $20 \times 20$ pixels that fully encloses the object. The IoU in this case will be 0.25 , which is eliminated from the threshold of 0.5 . Then, the third factor may be the loss function of YOLO, which treats errors the same in all sizes of bounding boxes, big or small ( $\mathrm{Du}, 2018)$. A small error in a large box is generally negligible but a small error in a small box has a more significant effect on the IoU.

However, detection was successfully done when YOLOWEED was tested on an $864 \times 688$ resolution video. The detection was done at $416 \times 416$ network resolution of YOLOv3. Fig. 4 shows some of the screen captures of the weed detections, which occurred at detection speeds 22.7 FPS to 24.4 FPS with GeForce GTX 1060. This further proves the potential of YOLOv3 in real-time object detection in videos. This detection speed was possible because the Darknet framework in YOLOv3 utilizes the GPU. However, this is slightly slower compared to the detection speed of Czymmek et al., (2019) at $416 \times 416$ network resolution, having a detection speed of 40.3 FPS using NVIDIA GeForce $1080 \mathrm{Ti}$. Also, the detection speed may be significantly different if the detection process is done without a GPU. In the recent advances, the performance of YOLOv3 can be fully maximized with recently-developed embedded deep learning modules for UAVs such as the Jetson TX2, which has a 256-core NVIDIA Maxwell GPU.

For future studies, the proponent has several recommenddations for the improvement of YOLO-WEED in detecting smaller weed objects and for the use of YOLOv3 in detecting other crops.

\section{(1) Annotation}

First, the relative size of the ground truth objects should be enlarged by lowering the altitude (approximately 1 to $2 \mathrm{~m}$ ) for the UAV image acquisition. This removes the need for cropping the training image or video. However, the labeling tool used in this research had a limitation in labeling full-resolution images as it crops the images. In addition, it had no zooming feature, which increases the chance of human error in labeling. Thus, it is recommended to use other

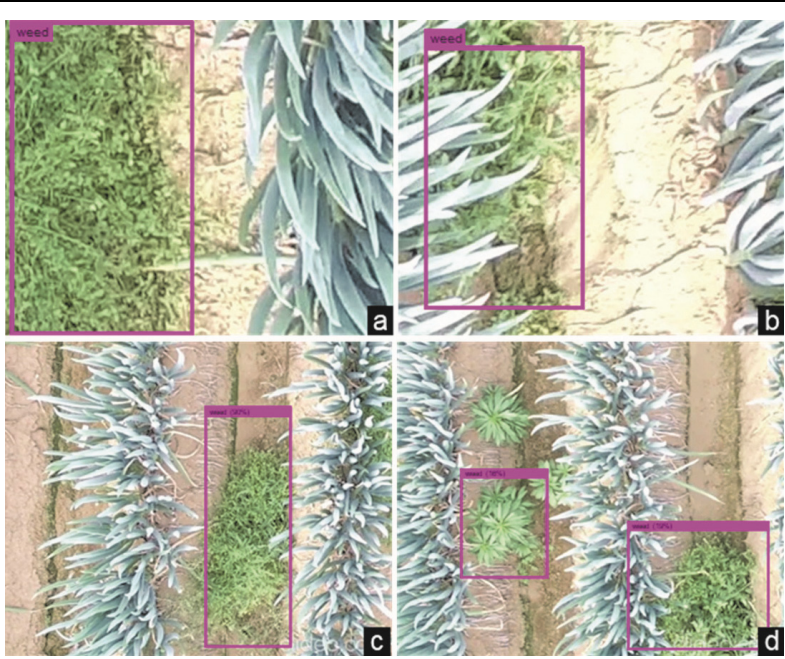

Fig. 4 (a) Test image with an instance of "weed" object is enclosed in a bounding box of $98 \%$ confidence at $24.8 \mathrm{FPS}$

(b) Test image with an occluded "weed" object was still detected at $85 \%$ confidence at 17 FPS

(c) An image frame from the tested $864 \times 688$ resolution video with instance of "weed" object detected at $90 \%$ confidence

(d) Another image frame from the tested video with smaller weeds detected at relatively lower confidence (16 and 19\%), including a false negative detection

labeling tools that have zooming features to reduce the human error in labeling small weeds. Second, an allowance of 5 pixels on average on each direction around small objects (less than $25 \times 25$ pixels) is recommended to be implemented, which is based on the method of ImageNet Large Scale Visual Recognition Challenge (ILSVRC) (Russakovsky et al., 2015). This will also reduce the effect of the loss function in amplifying the errors in small objects. Third, instance-level segmentation instead of bounding boxes as a form of annotation is recommended for a more accurate method of object detection and localization (Lin et al., 2014), especially for objects that are articulated. Finally, objects that are considered "difficult" to annotate based on the criteria of ILSVRC should be separated from the "weed" class (Russakovsky et al., 2015). These "difficult" objects are inevitable in training images and are often sources of bias in IoU calculations.

(2) Generalization ability

It is difficult to guarantee that the validation and test datasets in this study may be sufficient in representing the underlying unknown data distribution among weeds in green onion fields. This is because the source of the dataset was taken only from one clear day, from one field. This may lead to a weaker generalization ability once faced with unseen dataset. Data acquisition of different time and / or location is still recommended to be implemented conjointly with data augmentation in future studies. Also, despite being widely 
used in deep learning, the method of validation in this study may also have limitations identifying overfitting if the test data is not representative enough for unseen data (Zhang et al., 2020). Thus, it is recommended to explore other methods of validation that can detect a hidden overfitting problem, such as detecting overfitting through generation of adversarial examples from test data or similarly through comparison with a model trained with a perturbed version of the training data (Zhang et al., 2020).

\section{Conclusion}

This study developed and evaluated the performance of YOLO-WEED, a system that allows the detection of weeds in UAV video frames through the utilization of the deep learning algorithm YOLO. It demonstrated high speed (22.7 to 24.4 FPS), making it suitable for real-time weed detection for green onion fields in a single flight schedule to reduce time involvement for precision application of herbicides. Furthermore, it showed remarkable performance with test images, having a mean average precision of $93.81 \%$ and an F1 score of 0.94 . Lastly, YOLO-WEED performed fairly with the $864 \times 688$ resolution UAV video, having the limitation of YOLOv3 in detecting small objects minimized. Due to its higher speed and accuracy, in the future, the YOLO-WEED system can be potentially integrated into real-time automated aerial spray systems for green onion fields.

Having an on-board computer may be thought as an additional load for UAV sprayer systems. Fortunately, recently, small and lightweight embedded computing boards, such as NVIDIA's Jetson, have been developed for applications with weight/ power constraints. This makes embedded systems suitable for using YOLO-WEED for UAVs. A number of studies have tested the compatibility of YOLOv3 with aerial vehicles in different applications equipped with Jetson (Afifi et al., 2021; Montanari et al., 2018; Smolyanskiy et al., 2017; Yang et al., 2018). Therefore, embedded computing boards may pave the way for lighter and smarter UAV sprayer systems.

\section{Acknowledgements}

These individuals, who helped me attain the entirety of the study, are worthy of true recognition and appreciation: to my research supervisor, Associate Professor Tofael Ahamed, for sharing his insights and constructive criticisms, and for his constant support and guidance; to my colleagues who has assisted me in commencing my UAV flights and has given suggestions for the improvement of my research; To the TAG-MEXT program for providing me the financial support for my studies here in University of Tsukuba; to Associate Professor Nakao Nomura and Dr. Renato Reyes for giving me the opportunity to study in University of Tsukuba; and finally to my friends, family and partner who have given me moral support throughout my studies.

\section{References}

Afifi, M., Y. Ali, K. Amer, M. Shaker and M. ElHelw. 2021. Robust real-time pedestrian detection on embedded devices. Proceeding of the 13th International Conference on Machine Vision. 45. Rome, Italy, 2-6 November 2020. https://doi.org/10.1117/12.2587097, (Accessed 31 Mar. 2021).

Bargoti, S. and J. Underwood. 2017. Deep fruit detection in orchards. Proceedings of the 2017 IEEE International Conference on Robotics and Automation (ICRA). 3626-3633. Singapore, 29 May-3 June. https://doi.org/10.1109/ICRA.2017.7989417, (Accessed 9 Mar. 2021).

Czymmek, V., L. O. Harders, F. J. Knoll and S. Hussmann. 2019. Vision-based deep learning approach for real-time detection of weeds in organic farming. Proceedings of the 2019 IEEE International Instrumentation and Measurement Technology Conference (I2MTC). Auckland, New Zealand, 20-23 May. https://doi.org/10.1109/I2MTC.2019.8826921, (Accessed 9 Mar. 2021).

Du, J. 2018. Understanding of object detection based on CNN family and YOLO. Journal of Physics: Conference Series. 1004:012029. https://doi.org/10.1088/1742-6596/1004/1/012029, (Accessed 9 Mar. 2021).

Everingham, M., L. Van Gool, C. K. I. Williams, J. Winn and A. Zisserman. 2010. The pascal visual object classes (VOC) challenge. International Journal of Computer Vision. 88: 303-338. https:// doi.org/10.1007/s11263-009-0275-4, (Accessed 9 Mar. 2021).

FDA. 2016. Green Onions. USA: Food and Drug Administration. https://www.wifss.ucdavis.edu/wp-content/uploads/2016/10/Gre enOnions_PDF.pdf, (Accessed 9 Mar. 2021).

Gao, J., A. P. French, M. P. Pound, Y. He, T. P. Pridmore and J. G. Pieters. 2020. Deep convolutional neural networks for image-based Convolvulus sepium detection in sugar beet fields. Plant Methods. 16. https://doi.org/10.1186/s13007-020-00570-z, (Accessed 9 Mar. 2021).

Gilreath, J. P., B. M. Santos, P. R. Gilreath and D. N. Maynard. 2008. Efficacy of early post-transplant herbicides in leeks (Allium porrum L.). Crop Protection. 27: 847-850. https://doi.org/10.1016/ j.cropro.2007.06.005, (Accessed 9 Mar. 2021).

He, X. K., J. Bonds, A. Herbst and J. Langenakens. 2017. Recent development of unmanned aerial vehicle for plant protection in East Asia. International Journal of Agricultural and Biological Engineering. 10 (3): 18-30. https://www.researchgate.net/publication/ 317779797_Recent_development_of_unmanned_aerial_vehicle_ for_plant_protection_in_East_Asia, (Accessed 9 Mar. 2021).

Hewson, R. T. and H. A. Roberts. 1973. Some effects of weed competition on the growth of onions. Journal of Horticultural Science. 48 (1): 51-57. https://doi.org/10.1080/00221589.1973.11514506, (Accessed 9 Mar. 2021).

Krizhevsky, A., I. Sutskever and G. E. Hinton. 2017. ImageNet classification with deep convolutional neural networks. Communications of the ACM. 60 (6): 84-90. https://doi.org/10.1145/ 3065386, (Accessed 9 Mar. 2021).

Lan, Y., S. J. Thomson, Y. Huang, W. C. Hoffmann and H. Zhang. 
2010. Current status and future directions of precision aerial application for site-specific crop management in the USA. Computers and Electronics in Agriculture. 74 (1): 34-38. https://doi.org/10.1016/j.compag.2010.07.001, (Accessed 9 Mar. 2021).

Lin, T.-Y., M. Maire, S. Belongie, J. Hays, P. Perona, D. Ramanan, P. Dollár and C. L. Zitnick. 2014. Microsoft COCO: Common Objects in Context. Computer Vision - ECCV 2014, ed. Fleet, D., T. Pajdla, B. Schiele and T. Tuytelaars. 740-755. Switzerland: Springer International Publishing. https://doi.org/10.1007/978-3319-10602-1_48, (Accessed 9 Mar. 2021).

Montanari, A., F. Kringberg, A. Valentini, C. Mascolo and A. Prorok. 2018. Surveying areas in developing regions through context aware drone mobility. Proceedings of the 4th ACM Workshop on Micro Aerial Vehicle Networks, Systems, and Applications (DroNet'18). 27-32. Munich, Germany, 10-15 June. https://doi.org/ 10.1145/3213526.3213532, (Accessed 9 Mar. 2021).

Norsworthy, J. K., J. P. Smith and C. Meister. 2007. Tolerance of direct-seeded green onions to herbicides applied before or after crop emergence. Weed Technology. 21 (1): 119-123. https://doi. org/10.1614/WT-06-042.1, (Accessed 9 Mar. 2021).

Partel, V., S. Charan Kakarla and Y. Ampatzidis. 2019. Development and evaluation of a low-cost and smart technology for precision weed management utilizing artificial intelligence. Computers and Electronics in Agriculture. 157: 339-350. https://doi.org/10.1016/ j.compag.2018.12.048, (Accessed 9 Mar. 2021).

Qiu, S. and N. Shrivastava. 2017. BBox-Label-Tool. https:/github.com/ puzzledqs/BBox-Label-Tool, (Accessed 9 Mar. 2021).

Redmon, J. 2016. Darknet: Open Source Neural Networks in C. https://pjreddie.com/darknet/, (Accessed 9 Mar. 2021).

Redmon, J. and A. Farhadi. 2018. YOLOv3: An incremental improvement. arXiv:1804.02767. https://arxiv.org/abs/1804.02767, (Accessed 9 Mar. 2021).

Redmon, J. and A. Farhadi. 2017. YOLO9000: Better, faster, stronger. Proceeding of the 2017 IEEE Conference on Computer Vision and Pattern Recognition (CVPR). 6517-6525. Honolulu, HI,
USA, 21-26 July. https://doi.org/10.1109/CVPR.2017.690, (Accessed 9 Mar. 2021).

Russakovsky, O., J. Deng, H. Su, J. Krause, S. Satheesh, S. Ma, Z. Huang, A. Karpathy, A. Khosla, M. Bernstein, A. C. Berg and L. Fei-Fei. 2015. ImageNet large scale visual recognition challenge. International Journal of Computer Vision. 115: 211-252. https:// doi.org/10.1007/s11263-015-0816-y, (Accessed 9 Mar. 2021).

Smolyanskiy, N., A. Kamenev, J. Smith and S. Birchfield. 2017. Toward low-flying autonomous MAV trail navigation using deep neural networks for environmental awareness. Proceeding of the 2017 IEEE / RSJ International Conference on Intelligent Robots and Systems (IROS). 4241-4247. Vancouver, BC, Canada, 2428 September. https://doi.org/10.1109/IROS.2017.8206285, (Accessed 9 Mar. 2021).

Timmermann, C., R. Gerhards and W. Kühbauch. 2003. The economic impact of site-specific weed control. Precision Agriculture. 4: 249-260. https://doi.org/10.1023/A:1024988022674, (Accessed 9 Mar. 2021).

Tsouros, D. C., S. Bibi and P. G. Sarigiannidis. 2019. A review on UAV-based applications for precision agriculture. Information. 10 (11): 349. https://doi.org/10.3390/info10110349, (Accessed 9 Mar. 2021).

Yang, T., Q. Ren, F. Zhang, B. Xie, H. Ren, J. Li and Y. Zhang. 2018. Hybrid camera array-based UAV auto-landing on moving UGV in GPS-denied environment. Remote Sensing. 10 (11): 1829. https://doi.org/10.3390/rs10111829, (Accessed 9 Mar. 2021).

Zhang, J.M., M. Harman, L. Ma and Y. Liu. 2020. Machine learning testing: survey, landscapes and horizons. IEEE Transactions on Software Engineering. https://doi.org/10.1109/TSE.2019.2962027, (Accessed 31 Mar. 2021).

Zhang, R., C. Wang, X. Hu, Y. Liu, S. Chen and B. Su. 2018. Weed location and recognition based on UAV imaging and deep learning. International Journal of Precision Agricultural Aviation. 3 (1): 23-29. https://doi.org/10.33440/j.ijpaa.20200301.63, (Accessed 9 Mar. 2021).

(Received: 1 February 2020, Accepted: 23 August 2020) 